\title{
Mechanisms and modelling of creep in superalloys
}

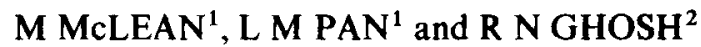 \\ ${ }^{1}$ Department of Materials, Imperial College of Science, Technology \& \\ Medicine, Prince Consort Road, London SW7 2BP, UK \\ ${ }^{2}$ National Metallurgical Laboratory, Jamshedpur 831007 , India
}

\begin{abstract}
The characteristics of creep deformation of nickel-base superalloys are reviewed and the implications for the micromechanisms controlling the behaviour are considered. The development of a model of the creep deformation that is compatible with the physical mechanisms is traced, first in an isotropic form and later incorporating full crystallographic anisotropy. The validity of the model and its ability to be extrapolated to more complex loading conditions are evaluated against a wide range of experimental measurements.
\end{abstract}

Keywords. Crystallographic anisotropy; nickel base superalloy; crystal rotation; shape change; low cycle fatigue; isotropic/anisotropic model; single crystal turbine blade.

\section{Introduction}

The development of nickel-base superalloys and the evolution of the gas turbine engine have been closely related, each having a history that stretches only about fifty years (Betteridge \& Shaw 1987). Indeed, the gas turbine is still by far the largest engine making use of these materials and the continual drive to improve engine efficiency has lead to very rapid changes in alloy composition and microstructure. More importantly, the technology pull aspect of the development has meant that new variants of superalloys have been very rapidly introduced into service.

A wide range of different alloy compositions and processing technologies have been developed for specific requirements in both different parts of gas turbines and for engines used for different applications. Thus, for example, in aero engines there is a requirement for materials with high creep strength capable of operating at high temperatures for use as turbine blades, whereas other materials with high yield and fatigue strengths capable of operating at high stresses and low temperatures are required for turbine disks. Similarly there are important differences in the requirements for materials for use in marine environments, for electricity generation and for civilian and military aircraft. However, alloys have been developed that suit all of these applications giving a wide range of compositions and processing routes. This has largely been achieved in an empirical manner and it is arguabie that the development of new nickel-based alloys is becoming increasingly difficult because of the maturity of the technology. 
Concentrating on the blade applications where creep behaviour is of particular importance we note that alloy microstructures have been controlled in addition to alloy composition in order to achieve optimum properties. The current state of the art for the most advanced turbine blade alloys is to produce these materials in single crystal form which leads to a high level of anisotropy of properties (Duhl 1987). Although the alloy strengths have probably been optimised to a level which is going to be difficult to exceed, it is unlikely that the full potential of these materials is yet being achieved. This is because there is as yet an inadequate understanding of the variation in relevant mechanical behaviour as a function of operating conditions and crystallographic anisotropy. Even if it were, the design procedures currently available cannot yet incorporate full time-dependent, anisotropic plasticity. Computer-aided design is developing very rapidly as computer power becomes cheaper and more readily available. However, these engineering developments can only be effective if there is a parallel improvement in the understanding and representation of material behaviour. Although the technology of alloy production is mature, engineering aspects of the use of single crystal superalloys are at a very early stage of development.

This paper will review current understanding of the factors influencing the creep behaviour of superalloys with particular emphasis on single crystal materials. It will describe the evolution of a model of anisotropic creep in single crystal superalloys that is expressed as constitutive laws which may be incorporated into finite element calculations of component behaviour. The paper will also indicate how the model has been validated and point to possible future directions.

\section{Creep behaviour of solid solutions and superalloys}

There is an extensive literature reporting studies of creep behaviour of a wide range of materials and of theoretical interpretation and modelling relating to these results (Wilshire \& Evans 1987, 1990, 1993). Concepts of the mechanisms of creep deformation have largely been derived from studies on simple metals and solid solution alloys, there is now a very good understanding of the micromechanisms controlling creep in these materials (Langdon 1985). However, there are some very significant differences in the creep behaviour of nickel-base superalloys compared to that of simpler metallic materials. To highlight a few of these differences:

- Plastic pre-strain of superalloys accelerates the creep rate whereas it hardens most solid solution alloys (Dyson et al 1976).

- There is very little difference in creep curves of superalloys produced under constant load and constant stress conditions whereas these tests give large differences in most solid solution alloys (Loveday \& Dyson 1990).

- Nickel-based superalloys exhibit very little, indeed probably no, steady state creep but are dominated by a tertiary creep regime of increasing creep rate. Solid solution alloys generally have an extensive steady state regime.

- Tertiary creep in nickel-base superalloys is exhibited in conditions of both tensile and compressive loading indicating that it cannot be due to cracking or cavitation. In solid solution alloys tertiary creep is generally associated with cavitation damage and is only apparent when there is a significant component of tensile loading (Tilley \& Harrison 1973). 
In view of these important differences it is essential to reconsider the mechanisms controlling creep deformation in multi-phase systems such as superalloys. The conventional models of recovery-controlled creep leading to a steady state behaviour that evolves into a tertiary regime when cavitation develops describes simple metals very well. However, they are not appropriate for superalloys, or indeed for many other microstructurally complex alloys. Dyson \& McLean (1983), Henderson \& McLean (1983), Dyson (1988) and Ion et al (1986) have, over a number of years, developed a model which attributes the characteristic softening of nickel-based superalloys to a progressive increase in the density of mobile dislocations that accumulate with increasing plastic strain. This can be thought of as being the result of inhibition of extensive glide of dislocations that encounter particles of the gamma prime precipitate. Insufficient dislocations are produced to provide a significant level of dislocation hardening and most new dislocations created remain mobile. Other factors that can contribute to the characteristic tertiary creep, such as loss of internal load bearing area due to the development of grain boundary cavities or reduction in load bearing cross-section during deformation (Ashby $\&$ Dyson 1984), which are common to most other materials, are relatively unimportant in single crystal superalloys and will not be considered here.

It has been possible to develop a relatively simple set of equations based on these concepts that adequately describe the creep behaviour of isotropic nickel-based alloys. More recently Ghosh et al (1990) and Pan et al (1993) have extended the approach to account for the material anisotropy in single crystal superalloys. This has taken the isotropic model as its starting point but recognises that even at high temperatures deformation results from dislocations moving on a few slip systems of well-defined crystallinity. Consequently, the model has been reframed in term of shear stresses and shear strains on these allowed slip systems and the total anisotropic deformation has been computed by summing the shear displacements from all active slip systems. This paper will briefly review the model and will compare its predictions with a range of validatory experiments.

\section{Isotropic model}

The model has been expressed in the formalism of continuum damage mechanics (Kachanov 1958) in which the creep rate $\dot{\varepsilon}$ is written as a function of state variables (or damage parameters) that represent the current condition of the material, in particular of the structural and microstructural features that control the strength of the material. If it is possible to derive equations describing the evolution of these state variables $\left(S_{1}, S_{2}, S_{3}\right)$ then the creep behaviour can be determined by integration of a set of coupled differential equations:

$$
\begin{aligned}
\dot{\varepsilon} & =f\left(\sigma, T, S_{1}, S_{2}, \ldots\right), \\
\dot{S}_{1} & =g\left(\sigma, T, S_{1}, S_{2}, \ldots\right), \\
\dot{S}_{2} & =h\left(\sigma, T, S_{1}, S_{2}, \ldots\right)
\end{aligned}
$$

The problem is to identify the explicit forms of these equations that are compatible with the known micromechanisms of deformation so that these can be evaluated quantitatively from standard creep data to allow extrapolation to complex loading conditions. 

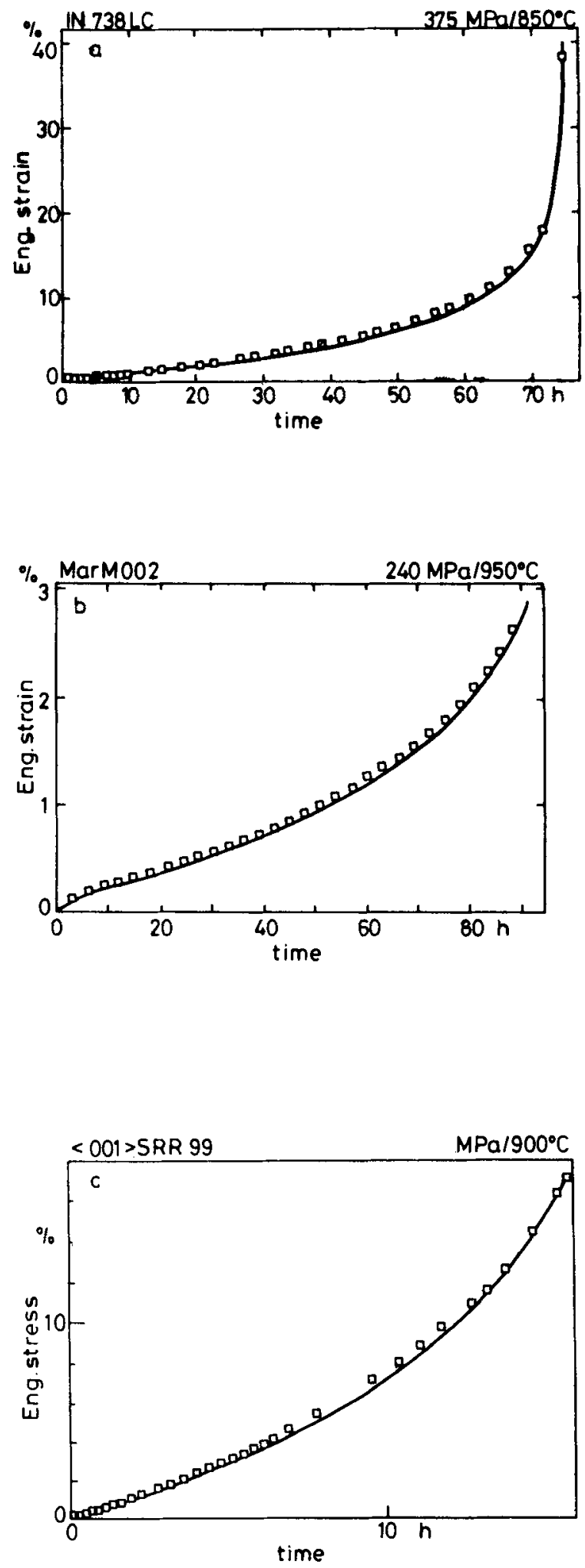

Figure 1. Examples of the fit of the isotropic model to creep curves for three different nickel base superalloys. 
Dyson \& McLean (1990) have given a comprehensive review of the isotropic model, so only the principal point will be given here. When cavitation and change of external material cross section can be neglected, the isotropic creep behaviour can be represented by a set of three equations involving two state variable $S, \omega$ and four constants $\dot{\varepsilon}_{i}, H, S_{\mathrm{ss}}, C$.

$$
\begin{aligned}
& \dot{\varepsilon}=\dot{\varepsilon}_{i}(1-S)(1+\omega), \\
& \dot{S}=H \dot{\varepsilon}_{i}\left(1-\left(S / S_{\text {SS }}\right)\right), \\
& \dot{\omega}=C \dot{\varepsilon} .
\end{aligned}
$$

$S$ is a dimensionless representation of an internal stress that progressively increases to a steady state value $S_{\mathrm{ss}}$ leading to primary creep. The increasing density of mobile dislocations $\rho$ is expressed through $\omega=\left(\rho-\rho_{i}\right) / \rho_{i}$ where the subscript $i$ indicates the initial value.

Dyson \& McLean (1990) describe a procedure for analysing a creep curve to evaluate the constants $\dot{\varepsilon}_{i}, H, S_{\mathrm{Ss}}, C$ and this has been successfully applied to severalmaterials and been shown to be particularly appropriate to nickel-base superalloys. Figure $1 \mathrm{com}$ pares several creep curves for nickel-base superalloys with the curves generated using equation set (2). This is not a particularly demanding test of the model since other more empirical approaches can provide equally accurate fitting of the shapes of individual creep curves. However, the differential form of equation set (2) allows the creep strains that develop during variations in stress and/or temperature to be evaluated quite easily. Figure 2 compares the measured creep strain during cyclic loading with a prediction using model constants evaluated from constant stress tests.

Ghosh \& McLean (1992) have extended this model to incorporate a wider range of loading conditions. In particular they show that strain-controlled deformation can be taken into account by the addition of one further equation into equation set (2). This represents the contribution of stress relaxation during variations in applied stress. If we represent the total and elastic contributions to strain by $\varepsilon_{T}, \varepsilon_{E}$ respectively, while the creep component has no subscript, the additional equation is:

$$
\cdot \dot{\sigma}=E \dot{\varepsilon}_{E}=E\left(\dot{\varepsilon}_{T}-\dot{\varepsilon}\right) \text {. }
$$

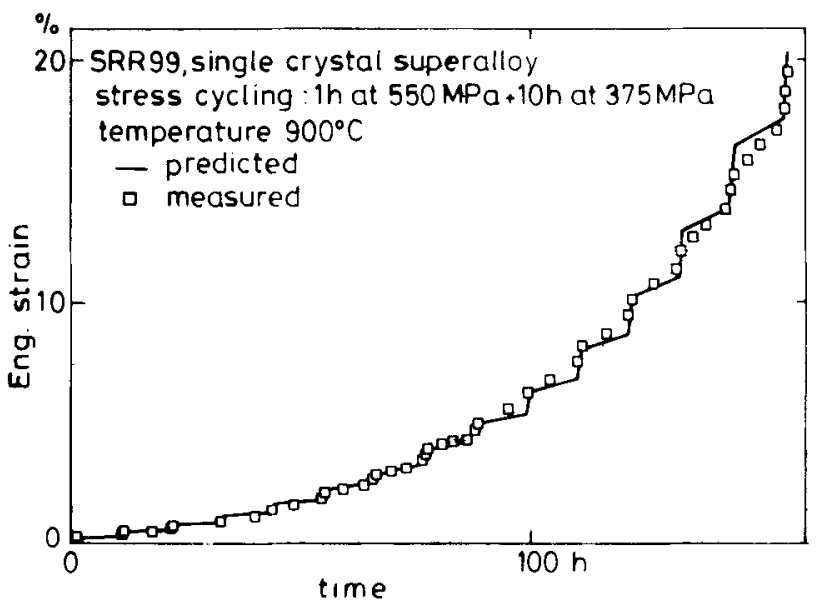

Figure 2. Comparison of the measured creep curves obtained under conditions of cyclic creep for the single crystal superalloy SRR99 axially stressed along the $\langle 001\rangle$ direction with the model prediction based on a database of constant stress creep tests. 

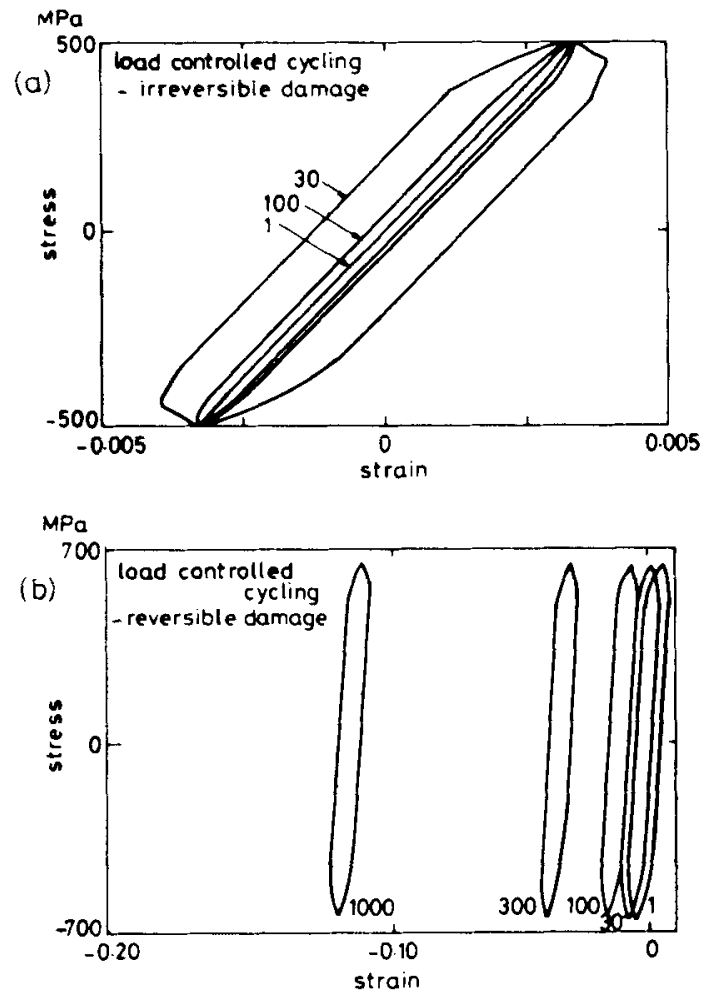

Figure 3. Predictions of the low cycle behaviour of IN738LC during constant load cycles based on model parameters established from a database of constant stress creep tests. The two predictions are for simulations of (a) irreversible growth of damage and (b) for damage that grows in tensile stresses but heals in compressive stresses.

Integration of the combination of equation set (2) and (3) subject to appropriate boundary conditions allows the simulation of several types of axial deformation: constant strain rate, stress relaxation, strain or load controlled low cycle fatigue. Ghosh \& McLean (1992) considered inter alia the consequences of reversible and irreversible damage; i.e. damage that grew under tension but healed under compressive loads, compared to damage that continued to grow irrespective of the direction of loading. Figure 3 illustrates the different predictions for these two types of damage for load-controlled LCF. For irreversible damage the plastic strain range progressively increases, while for reversible damage the strain range remains constant but the loops are progressively displaced giving a ratcheting of the material.

\section{Anisotropic model}

Extension of the model to account for anisotropic creep of single crystals by Ghosh et al (1990), and Pan et al (1993) considers deformation to be restricted to specific slip systems and to occur at a rate $\dot{\gamma}$ that depends on the resolved shear stress $\tau$, temperature $T$ and equivalent state variables to those introduced in the isotropic model. For the $k$ th slip system:

$$
\begin{aligned}
& \dot{\gamma}^{k}=\dot{\gamma}_{i}^{k}(1-S)(1+\omega) \\
& \dot{S}=H \dot{\gamma}_{i}^{k}\left(1-\left(S / S_{\text {ss }}\right)\right) \\
& \dot{\omega}=\beta \dot{\gamma}^{k}
\end{aligned}
$$


The total displacement $\varepsilon_{i j}$ is obtained by summing all $N$ components of shear on the allowed slip directions $\left\{n_{1} n_{2} n_{3}\right\}\left\langle b_{1} b_{2} b_{3}\right\rangle$

$$
\varepsilon_{i j}=\sum_{k=1}^{N} \gamma^{k} b_{i}^{k} n_{j}^{k}
$$

The subscripts $i, j$ take values 1,2 or 3 representing the threecube axes and $k$ identifies one of the $N$ slip vectors being considered. Having constructed the strain matrix, anisotropic deformation is completely defined. An arbitrary crystal direction $\left[x_{1} x_{2} x_{3}\right]$ will transform to a new orientation $\left[X_{1} X_{2} X_{3}\right]$ in the small strain approximation:

$$
\left[\begin{array}{l}
X_{1} \\
X_{2} \\
X_{3}
\end{array}\right]=\left[\begin{array}{ccc}
1+\varepsilon_{11} & \varepsilon_{12} & \varepsilon_{13} \\
\varepsilon_{21} & 1+\varepsilon_{22} & \varepsilon_{23} \\
\varepsilon_{31} & \varepsilon_{32} & 1+\varepsilon_{33}
\end{array}\right] \cdot\left[\begin{array}{l}
x_{1} \\
x_{2} \\
x_{3}
\end{array}\right] .
$$

Strain in that direction is given by the magnitudes of the initial and final vectors, $\varepsilon=(\bar{X}-\bar{x}) / \bar{x}$. If the constants of the creep equation are known, this allows the calculations of:

- crystal rotations during creep;

- strain in any direction;

- changes in material shape during deformation.

There is a considerable body of evidence in the literature that dislocation activity in nickel-base superalloys is largely restricted to octahedral planes even at high temperature (Caron et al 1988). There have also been reports of cube slip, in addition to elements of octahedral to cube cross-slip. In our analysis we allow the possibility of activity on $\{111\}\langle\overline{1} 10\rangle$ and $\{100\}\langle 011\rangle$. We have discounted the possibility of a $\{111\}\langle\overline{2} 11\rangle$ system for the reasons detailed by Ghosh et al (1990).

For certain orientations which are symmetrically oriented with respect to the active slip vectors there is no change in crystal orientation or of specimen shape as a result of deformation. In these cases the tensile and shear formulations of the model are totally equivalent. Moreover, for axial loading along $\langle 001\rangle$ there is no resolved shear stress on the cube planes, so deformation only results from octahedral slip. This allows the parameters of equation set (4) relating to $\{111\}\langle\overline{1} 10\rangle$ deformation to be evaluated from a creep database of $\langle 001\rangle$ specimens using simple geometrical transformations of the parameters of the tensile formulation. Similarly, the parameters for cube slip can be determined by analysis of $\langle 111\rangle$ specimens.

Figure 4 shows the optimum fit of the model to a creep curve for a $\langle 001\rangle$ specimen tested in tension under constant stress conditions; the tensile and shear formulations give identical predictions. The creep curve calculated for $\langle 111\rangle$ assuming that only octahedral slip is operative is also shown; this predicts a significantly longer life than for $\langle 001\rangle$ whereas a shorter life is measured as shown. The model also provides a good representation of $\langle 111\rangle$ creep curves by establishing the parameters for cube shear creep.

Having established the model parameters for both octahedral and cube shear creep as functions of stress and temperature by analysis of creep databases for $\langle 001\rangle$ and <111) specimens respectively it is straightforward to calculate the deformation that results for arbitrary crystal orientations and applied stresses.

The model can also be combined with a representation of the anisotropy of elastic constants to include aspects of stress relaxation needed to account for arbitrary loading 


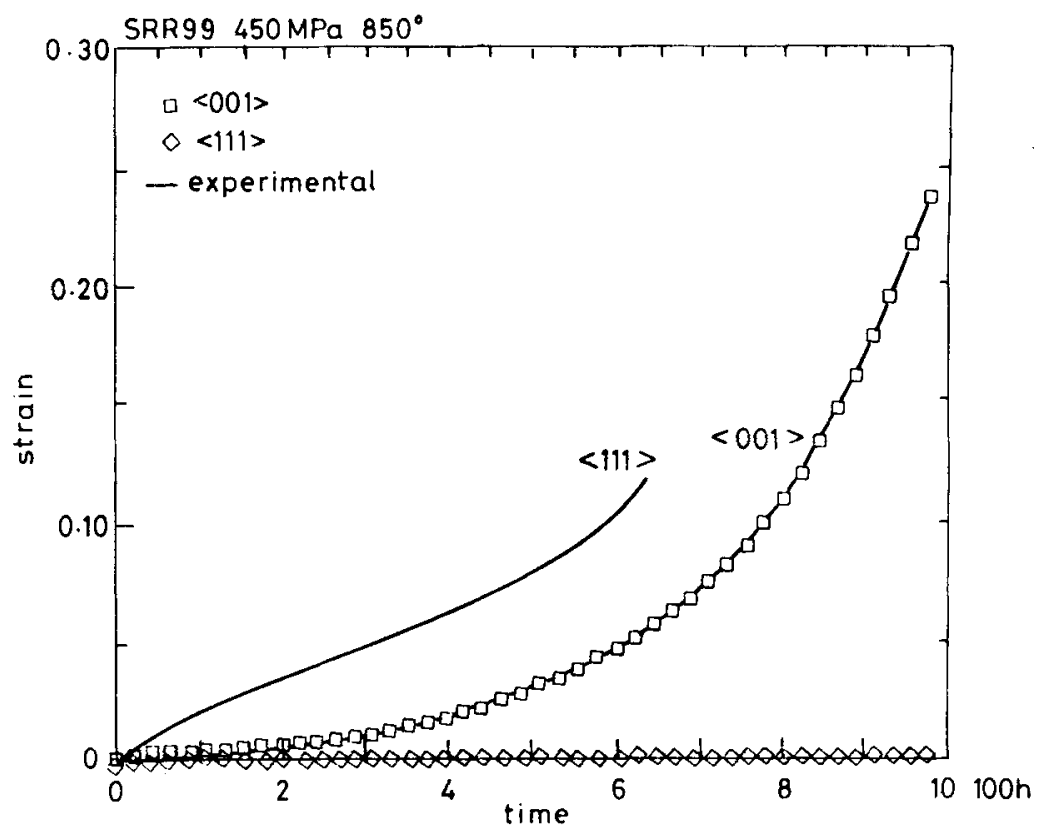

Figure 4. Comparison of the experimental and calculated creep curves for $\langle 001\rangle$ and $\langle 111\rangle$ oriented specimens of SRR 99 tested at $450 \mathrm{MPa} / 850^{\circ} \mathrm{C}$ assuming that only $\{111\}\langle 110\rangle$ glide operates.

conditions and arbitrary orientations (Pan et al 1993). For axial loading along the $\langle h k l\rangle$ direction the Young's modulus can be expressed in terms of the components of the compliance tensor $S$.

$$
E_{\langle h k l\rangle}=\left[S_{11}+\left\{S_{44}-2\left(S_{11}-S_{12}\right)\right\}\left(h^{2} k^{2}+k^{2} l^{2}+l^{2} h^{2}\right)\right]^{-1} .
$$

Using a time-stepping sequence, the creep strain in the $\langle h k l\rangle$ is computed and combined with the equation to determine the stress under strain-controlled conditions.

$$
\sigma=E_{\langle h k l\rangle}\left(\varepsilon_{T}-\varepsilon\right)
$$

\subsection{Validation of anisotropic model}

The model can provide an acceptable representation of a database of uniaxial creep results for the symmetrical $\langle 001\rangle$ orientation, as indicated in figure 5 , but this is not a particularly demanding test since equally good fits can be obtained by empirical models. The more demanding tests are to assess the ability of the model to extrapolate successfully from creep data for simple orientations to predict:

- creep behaviour for arbitrary complex orientations being able to account for variations in the form and magnitude of the anisotropy of creep as functions of stress and temperature;

- changes in orientation with creep strain;

- shape distortions of creep test specimens during deformation; 


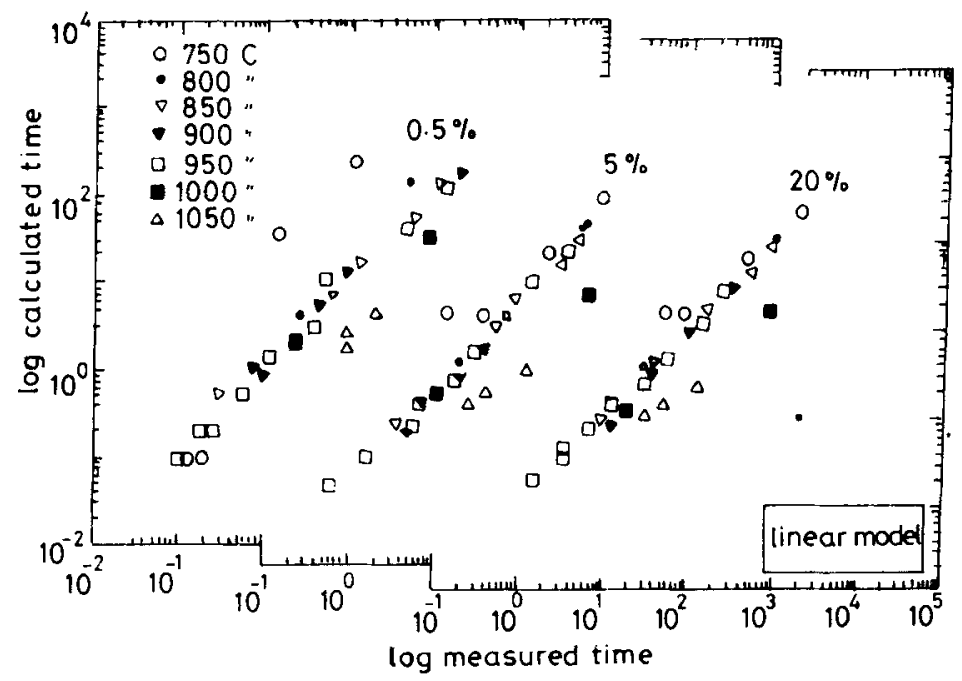

Figure 5. Comparison of the measured and calculated times to (a) $0.5 \%$, (b) $5 \%$ and (c) $25 \%$ elongations for SRR99.

- material response to a range of stress and strain controlled deformation for both simple and complex orientations.

All these aspects have been evaluated experimentally.

4.1a Anisotropy of creep: Figures 6a-c show the predicted and measured tensile creep curves for $\langle 001\rangle$ and $\langle 111\rangle$ orientations of the alloy SRR99 for three different stresses at $850^{\circ} \mathrm{C}$ in which the observed changes in relative creep strength are successfully reproduced bearing in mind the intrinsic scatter in creep data (Ghosh et al 1990). It is also possible to calculate the creep curves for arbitrary orientations but this is not a particularly demanding test.

4.1b Orientation changes: Uniaxially stressed specimens of simple symmetric orientations are not predicted to exhibit any change in orientation during creep deformation, and none are observed. However, in the case of complex orientations and/or multiaxial stresses where some slip systems are disproportionately activated there can be an expectation of quite large lattice rotations. Unfortunately, the increase in dislocation density with accumulating creep strain makes the accurate determination of crystal orientation by conventional X-ray techniques extremely difficult. Electron back-scatter patterns produced in a scanning electron microscope gives precise orientations with high spatial resolution (Dingley 1984). A series of fractured creep specimens, all of which exhibited a considerable degree of necking, have been examined using this technique to relate the change in orientation along the specimen length to the local strain as measured by the reduction in area. Figure 7 compares the measured changes in the axial orientation with those calculated by the model using parameters established from the creep database for $\langle 001\rangle$ and $\langle 111\rangle$ orientations (McLean et al 1992). There is clearly a high level of agreement between prediction and measurement of rotations that do not correlate with those predicted by the operation of a single slip system. 
(a)

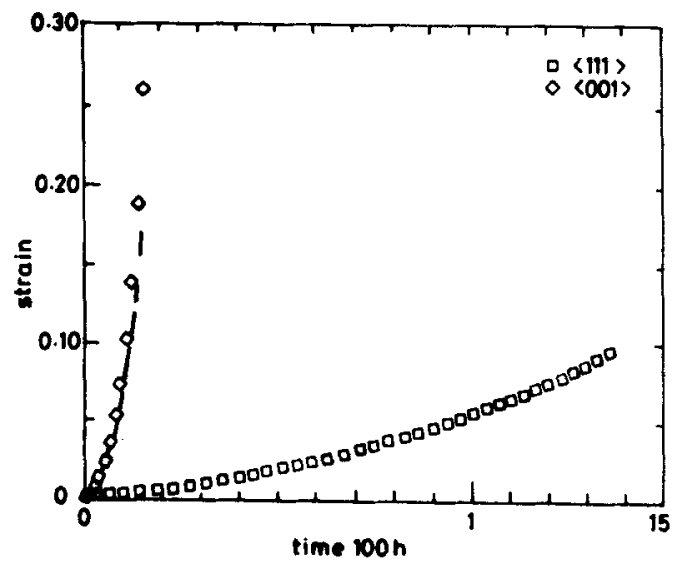

(b)

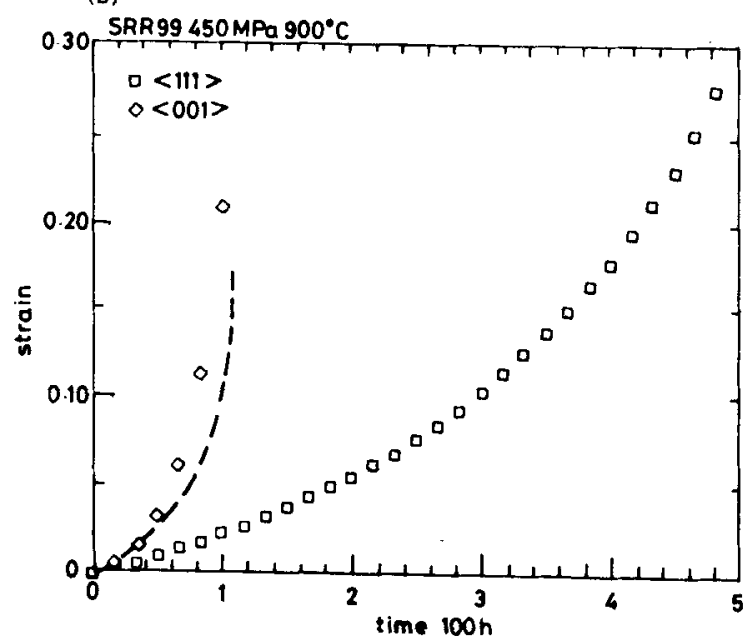

(c)

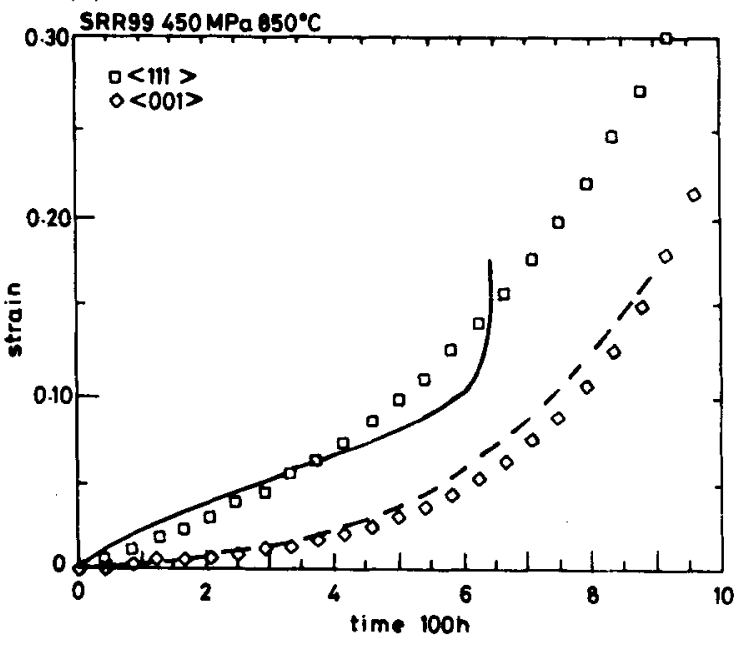

Figure 6. Measured and calculated creep curves for SRR99 with $\langle 001\rangle$ and $\langle 111\rangle$ orientations tested in tension at various temperatures $(850,900$ and $950^{\circ} \mathrm{C}$ ) and a stress of $450 \mathrm{MPa}$ showing the variation in relative creep performance. 


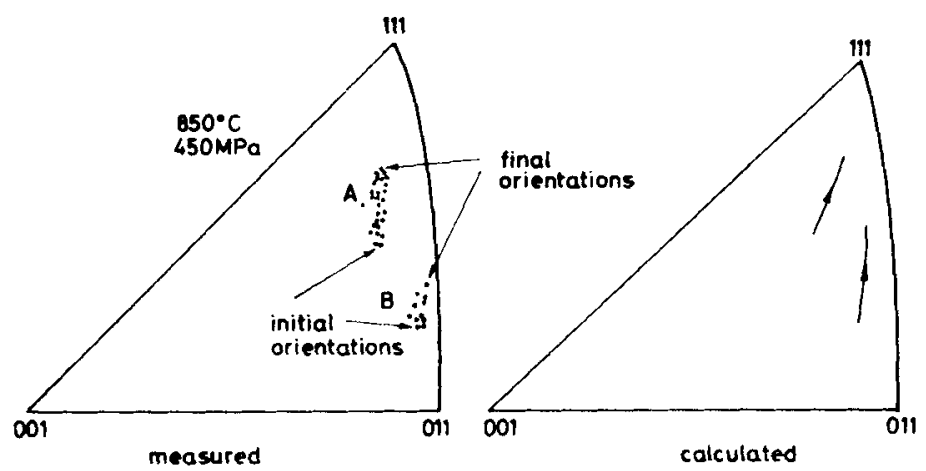

Figure 7. Crystal rotations during creep deformation of SRR99 of different complex orientations tested at $850^{\circ} \mathrm{C} / 450 \mathrm{MPa}$ : (a) experimental measurements using electron back-scatter patterns and (b) model predictions.

4.1c Shape changes: The diameters of a series of creep specimens were accurately determined as functions of specimen rotation and reduction in area along the length of the necked specimens, carefully correlating the measurements with the crystallographic orientation of the specimens (Pan et al 1994, 1995). The measured and calculated cross-sections are shown in figure 8 as opposite halves of the same figure since planar symmetry is expected. Clearly, for the example shown, there is close agreement between measurements and model prediction for the orientation shown. In this case the model predicts a predominance of cube slip. Indeed these measurements combined with those of the orientation changes provide incontrovertible evidence of shear on cube planes on a macroscopic scale. This does not necessarily imply that there is extensive dislocation activity on cube planes at a microscopic level.
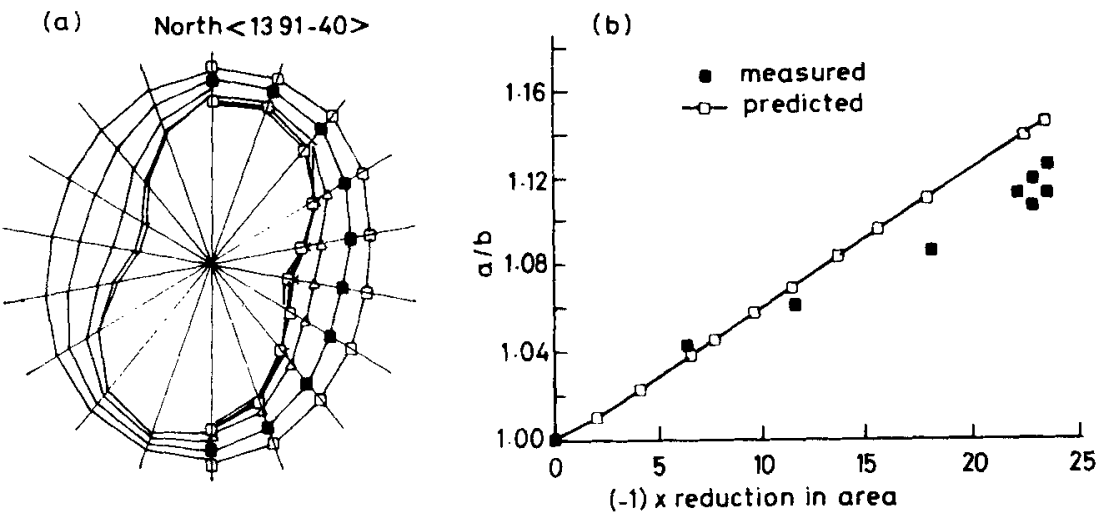

Figure 8. Comparisons of measured and predicted cross-sections of creep specimens of SRR99 at various reductions in area along the necked test-piece: (a) composite polar plots showing measurements (right) and calculations (left) and (b) ratio of maximum/minimum diameters as a function of reduction in area. 
(a)
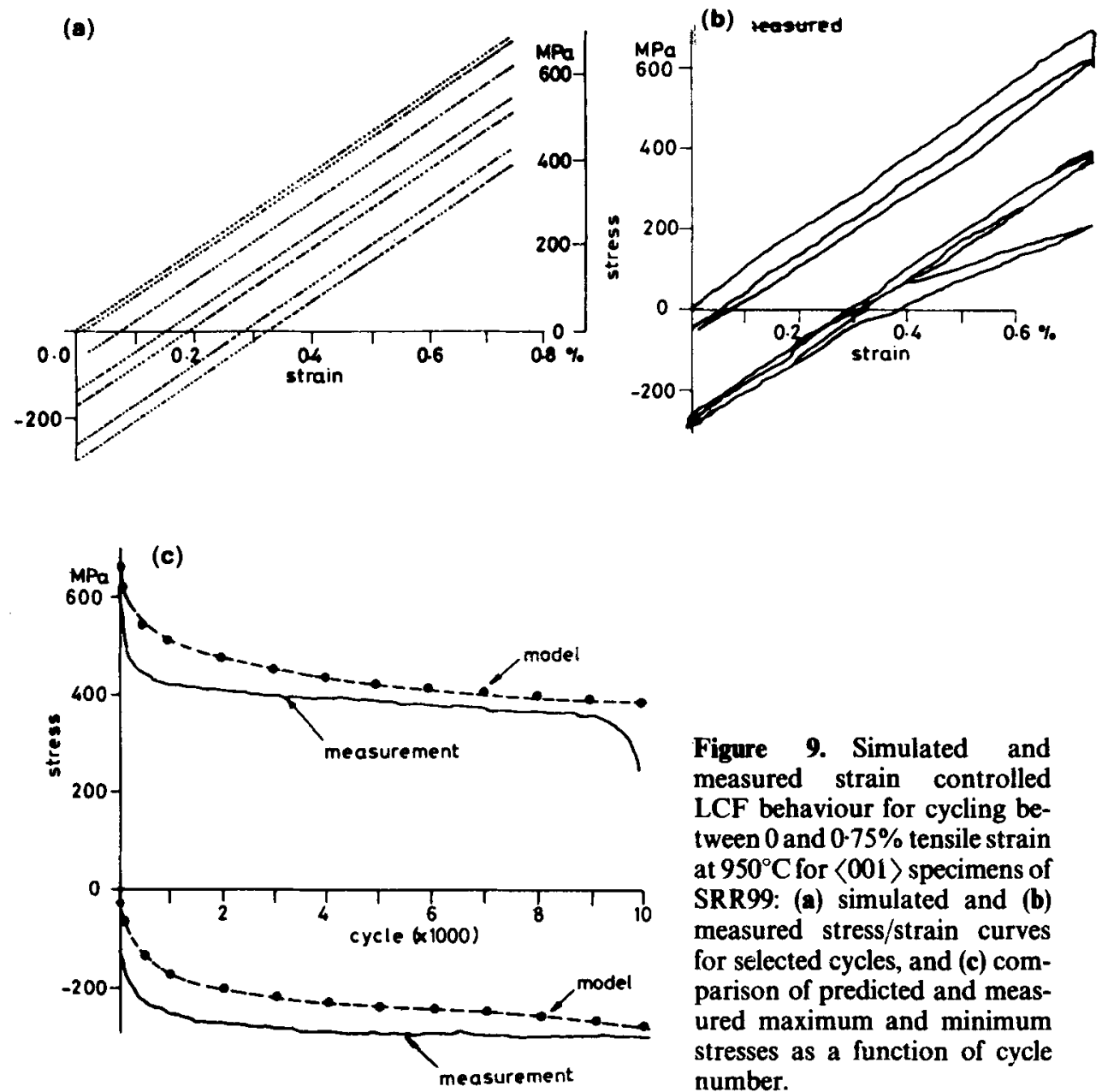

Figure 9. Simulated and measured strain controlled LCF behaviour for cycling between 0 and $0.75 \%$ tensile strain at $950^{\circ} \mathrm{C}$ for $\langle 001\rangle$ specimens of SRR99: (a) simulated and (b) measured stress/strain curves for selected cycles, and (c) comparison of predicted and measured maximum and minimum stresses as a function of cycle number.

4.1d Low cycle fatigue: The results of a series of high strain, elevated temperaturelow cycle fatigue tests for SRR99 have been compared with simulations using model parameters derived from a creep database (Pan et al 1994). A typical example, for strain controlled cycle between strain limits of 0 and $0.75 \%$ tensile strain at $950^{\circ} \mathrm{C}$, is shown in figure 9. Each cycle taken individually exhibits largely elastic deformation, but there is a shakedown of stresses reducing the maximum tensile stress and placing the unloading cycle into compression. This is consistent with there being some relaxation of the elastic tensile stress due to creep. The model calculations reproduce the observed behaviour to an acceptable degree of accuracy, particularly in view of the large difference in strain rates associated with the low cycle fatigue tests and with the creep tests forming the database.

\section{Conclusions}

A physics-based model of creep deformation in nickel-base superalloys has been developed, first in an isotropic form and later to account for the full crystallographic 
anisotropy of single crystal superalloys, that successfully represents the principal characteristics of creep in these materials. The model has been validated against both direct creep data and a number of more indirect measures of creep deformation. Comparisons of measurements with model predictions of orientation and shape changes and of the effects of cyclic loading provide particularly demanding tests of the model that are successfully achieved.

Much of the work described in this review has been funded by the Defence Research Agency and creep data and specimens for examination were supplied by Dr M R Winstone of DRA.

\section{References}

Ashby M F, Dyson B F 1984 Creep damage mechanics and micromechanisms. Advances in fracture research (eds) S R Valluri, D M R Taplin, P Rama Rao, J F Knott, R Dubey (Oxford: Pergamon) vol. 1, pp. 3-30

Betteridge W, Shaw S W K 1987 Development of superalloys. Mater. Sci. Technol. 3: 682-694

Caron P, Ohta Y, Nakagawa Y G, Khan T 1988 Superalloys (eds) D N Duhl et al (Warrendale, PA: The Metallurgical Society) p. 215

Dingley D J 1984 Proc. Conf. "Scanning Electron Microscopy - II" Chicago, p. 569

Duhl D N 1987 Superalloys - II (eds) C T Sims et al (New York: Wiley)

Dyson B F, Loveday M S, Rodgers M J 1976 Grain boundary cavitation under various states of applied stress. Proc. R. Soc. London A349: 245-259

Dyson B F, McLean M 1983 Particle coarsening, $\sigma_{0}$ and tertiary creep Acta Metall. Mater. 31: $17-27$

Dyson B F 1988 Creep and fracture of metals: Mechanisms and mechanics. Rev. Phys. Appl. 23: 605

Dyson B F, McLean M 1990 Creep deformation of engineering alloys: Developments from physical modelling. ISIJ 30: 802-811

Ghosh R N, Curtis R V, McLean M 1990 Creep deformation of single crystal superalloys modelling the crystallographic anisotropy. Acta. Metall. Mater. 38: 1977-1992

Ghosh R N, McLean M 1992 High temperature deformation in engineering alloys - Modelling for strain or load control. Acta. Metall. Mater. 40: 3075-3083

Henderson P J, McLean M 1983 Microstructural contribution to friction stress and recovery kinetics during creep of nickel-base superalloys IN 738 LC. Acta. Metall. Mater. 31: 1203-1219

Ion J C, Barbosa A, Ashby M F, Dyson B F, McLean M 1986 The modelling of creep for engineering design - I. NPL Report DMA A115, National Physical Laboratory, Teddington

Kachanov L M 1958 Time of the rupture process under creep conditions. Izv. Akad. Nauk. SSR 8: 26

Langdon T E 1985 In Dislocations and properties of real materials, Book No. 323 (London: The Institute of Materials) p. 221

Loveday M S, Dyson B F 1990 In Proc. 4th Int. Conf. on Creep and Fracture of Engineering materials and structures (London: The Institute of Materials) p. 947

McLean M, Ghosh R N, Curtis R V, Basu-Conlin U, Winstone M R 1992 Anisotropy of high temperature deformation of single crystal superalloys. In Superalloys (eds) S Antolovitch et al (Warrendale, PA: The Metallurgical Society) pp. 609-618

Pan L M, Ghosh R N, McLean M 1993 Extension of an anisotropic creep model to general high temperature deformation of a single crystal superalloy. Proc. of EuroMat 93, Paris (to be published) 
Pan L M, Ghosh R N, McLean M 1994 Load and strain controlled deformation of a single crystal superalloy-Modelling and validation. Proc. of ICSMA 6, Sendai, Japan (The Japanese Institute of Metals) pp 583-586

Pan L M, Scheibli I, Shollock B A McLean M 1995 Asymmetric creep deformation of a single crystal superalloy. Acta Metall. Mater. 43: 1375-1384

Tilley G P, Harrison G F 1973 J. Strain Anal. 8: 124

Wilshire B, Evans R W (eds) 1987, 1990, 1993 Proc. Swansea conferences on creep and fracture of engineering materials and structures (London: Institute of Materials) 